\title{
Does involvement in a cohort study improve health and affect health inequalities? A natural experiment
}

\author{
Annie Quick ${ }^{1 *}$, Jan R. Böhnke ${ }^{2}$, John Wright ${ }^{3}$ and Kate E. Pickett ${ }^{1}$
}

\begin{abstract}
Background: Evidence suggests that the process of taking part in health research can improve participants' health, independent of any intended intervention. However, no research has yet explored whether these effects differ across socioeconomic groups. If the effect of mere participation in health research also has a social gradient this could increase health inequalities and bias research results. This study used the Born in Bradford family cohort (BIB) to explore whether simply taking part in BIB had improved participants' health and, if so, whether this effect was mediated by socioeconomic status.

Methods: Survey data on self-reported health behaviours were collected between 2007 and 2010 as part of BIB. These were augmented by clinical data on birth weight. Pregnant women on their second pregnancy, joining BIB for the first time formed the control group. Their health was compared to women on their second pregnancy who had both pregnancies within the study, who formed the exposed group. In order to limit the inherent bias in a non-randomised study, propensity score analysis was used, matching on age, ethnicity, education and date of questionnaire. The results were then compared according to mothers' education.
\end{abstract}

Results: Of six outcomes tested, only alcohol consumption showed a statistically significant reduction with exposure to $\mathrm{BIB}$ (OR: 0.35, 95\% Cls 0.13, 0.92). Although effect estimates were larger for women with higher education compared to lower education, these effects were not statistically significant.

Conclusions: Despite one significant finding, these results overall are insufficient to conclude that simply taking part in BIB affected participants' health. We recommend that socioeconomic status is considered in future studies testing effects of research participation, and that randomised studies with larger sample sizes are conducted.

Keywords: Born in Bradford, Hawthorne effect, Health inequalities, Mere measurement

\section{Background}

Researchers have long been interested in whether the experience of being part of a research study can change the behaviour of participants. When these changes are due purely to the experience of being in a study - rather than as a result of any intervention - this is sometimes called a Hawthorne effect, measurement reactivity, or mere measurement effect [1-3]. Such an effect might occur as a result of filling in a questionnaire, being observed by a researcher, or taking part in an interview.

\footnotetext{
* Correspondence: annie.quick@neweconomics.org

'Department of Health Sciences, University of York, Heslington, York YO10 5DD, UK

Full list of author information is available at the end of the article
}

This behaviour change might be the result of increasing participants' awareness by asking about a health behaviour, or through prompting participants to reflect on their own choices.

Existing research suggests that being involved in a research study can produce statistically significant, though generally small effects in participants' health behaviours. A recent meta-analysis examined the effect of asking questions on participants' behaviour [4]. From 41 studies the authors found a small overall change in behaviour (Standardized Mean Difference $=0.09$; 95\% CI $[0.04,0.13] ; \mathrm{k}=33$ ) for those who had answered questionnaires or surveys compared to participants with no measurement, or those with other forms of measurement. 
However, studies did show publication bias, suggesting the effect size may be overestimated.

Another meta-analysis examined the mere measurement effect on a more homogenous sample of studies: randomised controlled trials of brief interventions for alcohol consumption [5]. The review identified eight nested trials carried out to assess the impact of measurement on alcohol use independent of the intervention. The meta-analysis did not find a statistically significant effect $(p=0.053)$, although the authors highlighted that the effect size was relatively large (those who had been measured at baseline drank 1.5 fewer units a week than those who had not), and statistical power was low. These randomised studies are particularly valuable as they avoid a number of biases included in observational studies, and more such studies would help to increase the power for meta-analyses and provide a fuller picture of the role of mere measurement.

In a narrative review, French and Sutton [2] found evidence of small, but significant effects from mere measurement. For example, one study was a cluster randomised controlled trial of physical activity promotion in which participants in the control and intervention groups were further randomised either to have measurements taken at baseline, eight weeks and six months, or just at six months. The measurement was a thirteen-page questionnaire, and height, weight and waist circumference were also measured at baseline. The number of people meeting the recommended exercise level was $50 \%$ higher in those who filled out the questionnaire three times, compared to those who filled it out once (OR: 1.5, 95\% CI: 1.10, 2.06). No significant association was found, however, for the proportion of participants spending at least $150 \mathrm{~min}$ per week on physical activity [6].

Using patient information gathered via questionnaires is popular both in the areas of practice oriented research in psychotherapy [7] as well as in more general health contexts in order to collect patient reported outcomes [8]. In these settings repeated assessments during the course of care are obtained from the individual patients and generally these assessments, if fed back to clinicians, have been shown to improve treatment outcomes especially for patients that were on a trajectory of stagnation or even deterioration $[9,10]$. Whether this effect is due to changes in practice or just the repeated assessments is a topic of current debate [11-13].

Many of the health behaviours examined in studies of mere measurement have a social gradient, whereby people who are more advantaged in income or education have better health behaviours than those lower down the social ladder. These include behaviours such as physical activity [6] or smoking [1]. However, there is almost no evidence of how, if at all, mere measurement differentially affects people of different socioeconomic status (SES). Given that many of the studies of mere measurement are nested within larger studies it is likely that data on SES were available, but this was rarely studied. One exception was found: a randomised controlled trial by van Sluijs et al., measuring the effect of surveying physical activity, found a significant change in behaviour due to measurement, but no significant change in the model when adjusting for confounders, among which were employment and education [6].

Very little research has been carried out exploring the causal processes behind mere measurement, beyond a general raising of awareness [2]. It is possible that mere measurement could prompt behaviour change through similar processes as health promotion activities. Clearly, the comparison is not exact; unlike mere measurement, health promotion generally involves the provision of information or explicit suggestions for behaviour change. However, it has been shown that information on its own is often ineffective at changing behaviour [14]. In the absence of direct studies on mere measurement, then, the way in which health promotion interacts with SES was examined.

One systematic review explored the effect of health promotion and education campaigns according to SES [15]. Studies examining the effects of accident prevention schemes, educational books for pregnant women and immunisation found that people with higher SES improved more, so that the overall improvement was at the expense of widening health inequalities. In the US, Pickett et al. found that the widely commended 'Back to Sleep' campaign to prevent Sudden Infant Death Syndrome (SIDS) not only increased race and SES inequalities in rates of SIDS, but the odds ratio for SIDS associated with lower SES actually increased during the campaign [16].

The history of smoking is one example of the inequitable effects of health promotion. Although there was no clear social gradient in smoking in the first half of the twentieth century, a social gradient emerged with the 'Smoking Kills' campaigns [17, 18]. Smoking prevalence changed very little in the lowest social class quintile between 1973 and 1998, whereas all other quintiles saw significant reductions [19]. An evaluation of England's smoking cessation services in 2005 found a similar trend. Out of those smokers who took up smoking cessation services, disadvantaged groups had cessation rates of $8.7 \%$, compared to a rate of $17.4 \%$ in the most advantaged groups [20].

If mere measurement and health promotion affect health behaviours through similar causal pathways then the negative effects of health promotion on equality raise the question of whether mere measurement, too, may increase health inequalities. 
This study therefore aimed to address two sequential questions:

1. Does simply taking part in a research study improve participants' health behaviour?

2. If so, is this effect mediated by socioeconomic status?

\section{Methods}

\section{The Born in Bradford family cohort study}

Born in Bradford (BIB) is a longitudinal multi-ethnic family cohort study aiming to examine the impact of environmental, psychological and genetic factors on maternal and child health and wellbeing [20]. Bradford is a city in the North of England with high levels of deprivation and ethnic diversity. Approximately half of the births in the city are to mothers of South Asian origin. Women were recruited while waiting for their glucose tolerance test, a routine procedure offered to all pregnant women registered at the Bradford Royal Infirmary, at 26-28 weeks gestation. For those consenting, a baseline questionnaire was completed via an interview with a study administrator ${ }^{1}$.

The baseline questionnaire for the mothers was transliterated into Urdu and Mirpuri using a standardized process, so that words and phrases corresponded with the original English version. As Mirpuri does not have a written form trained bilingual interviewers administered the transliterated questionnaires to Mirpuri speakers.

The full BIB cohort recruited 12,453 women during 13,776 pregnancies between 2007 and 2010 and the cohort is broadly characteristic of the city's maternal population.

\section{Study design}

During the course of the cohort study some women became pregnant more than once. These women were invited to include their additional babies in the cohort and fill out another baseline questionnaire. This feature of the cohort was used to assess the effect of BIB on mothers' behaviour during pregnancy, as the health behaviours of mothers having their second baby in the cohort (who can be referred to as BIB
P2s) could be compared with the health behaviours of mothers enrolled into BIB for the first time (who can be referred to as BIB P1s), who had not yet been exposed to the study. In our sample, health behaviours differed according to parity ${ }^{2}$. For example, using data from all BIB P1s, the odds of smoking during pregnancy decreased with each additional child, with an adjusted odds ratio of 0.86 (95\% CIs $0.82,0.91 ; p<0.001$ ). We therefore restricted the study to include only women on their second pregnancies.

The study design is presented in Fig. 1. The control group is circled on the left hand side. These women had one pregnancy before BIB started, and a second child who was included in BIB. Their BIB P1 acts as our control. Their baseline questionnaire was filled out as they entered the study, so being part of the study should not have influenced their responses.

The exposed group is circled on the right hand side. These are women who had two children and both of them were included in the BIB study. They filled in the BIB questionnaire for each pregnancy. The data collected during their second pregnancy in the cohort acts as our exposed group because they have been exposed by filling out the BIB questionnaire during their first pregnancy (BIB P1). This study used only their responses from the questionnaire completed during their second BIB pregnancy. The data from their first BIB pregnancy was disregarded, so the control and exposed groups consist of different women.

\section{Outcomes and data collection Health behaviours}

The current official NHS advice is not to smoke, to consume only small quantities of caffeine during pregnancy and to take vitamin D supplements ${ }^{3}$. Women are also advised not to drink alcohol, although there is some leniency in the consumption of small quantities. Two measures for alcohol were therefore included to reflect differing levels of alcohol consumption. Five health behaviours were therefore analysed, all of which were dichotomous variables:

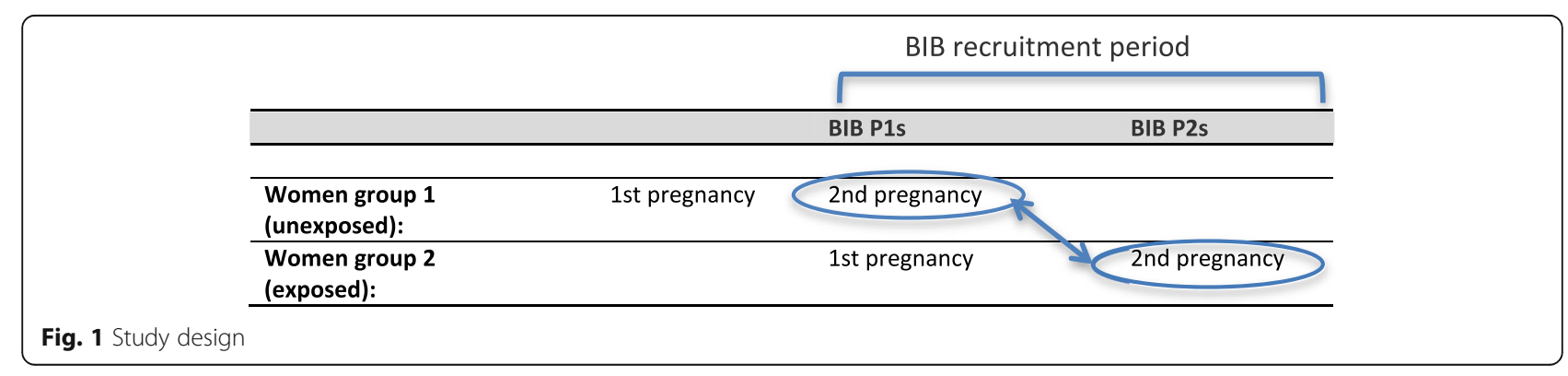


- Smoking. Women were coded yes if they reported smoking one or more cigarettes a day during pregnancy.

- Drinking any alcohol. Women were coded yes if they reported drinking any alcohol during the first three months of pregnancy.

- 5 or more units of alcohol once a month or more. Women were coded yes if they reported drinking five or more units of alcohol 1-3 times a month or more during the first three months of pregnancy.

- Caffeine consumption. To reflect NHS advice that consuming small quantities of caffeine is acceptable, women were coded yes if they reported drinking more than one caffeinated drink per day, during the previous four weeks.

- Vitamin D supplementation. Women were coded yes if they reported consuming vitamin D, Pregnacare (Vitabiotics) or Sanatogen prenatal (Bayer), which both contain vitamin $\mathrm{D}$, during the previous four weeks.

All five behavioural outcomes were collected as part of the BIB baseline questionnaire at 28 weeks gestation.

\section{Clinical outcome}

Given the potential biases in self-reporting, birth weight was also used as an objective measure to act as a check on the reliability of self-reported behaviours. Research suggests that the risk of low birth weight is increased by smoking [21], alcohol [22], and possibly caffeine [23]. There is also some evidence that vitamin $\mathrm{D}$ is preventative of low birth weight $[24,25]$.

Birth weight was a continuous variable and taken from the maternity IT system.

Missing data was negligible for all outcome variables (maximum $0.3 \%$ ).

\section{Covariates}

Estimates were adjusted for age in years, ethnicity (white British, Pakistani and other), mother's education $(<5$ GCSE's, 5 GCSEs, A Levels, higher than A Levels, other), and date of questionnaire.

The date of questionnaire was included because BIB P2s would occur, on average, later in the recruitment period than BIB P1s. A difference between BIB P1s and BIB P2s could therefore reflect changes in health behaviour in the population over time, rather than the effect of being part of BIB.

All covariates came from the BIB baseline questionnaire except mother's age, which was obtained from the maternity record. These covariates were chosen a priori, on the basis that they would be associated with either health behaviour outcomes, or with the likelihood of a mother having two pregnancies within the BIB recruitment period.

\section{Statistical methods}

Given the possibility of bias in the absence of randomisation, we used propensity score matching, which is increasingly recognised as a robust method for assessing exposure effects in a non-experimental study design [26].

The propensity score is the conditional probability of having been exposed, given a set of observed covariates [27]. In the current study, the propensity score was calculated based on a multiple logistic regression model predicting previous exposure to BIB. This way, each mother's propensity score represented the probability that she was a BIB P2 (rather than a BIB P1) based on her age, ethnicity, education, and the date she filled out her questionnaire.

Nearest neighbour matching with common support was then used to create matched pairs. This involved firstly matching each BIB P1 in our control group with a BIB P2 with the closest propensity score. If there was more than one BIB P1 with an identical propensity score, one was chosen at random. Secondly, common support was applied, whereby BIB P2s whose propensity score was higher or lower than the range in propensity scores of the BIB P1s, and BIB P1s with a propensity score higher or lower than all BIB P2s were all excluded, ensuring that both groups had propensity scores within the same range. All the other BIB P1s were then dropped from the analysis to leave only the matched pairs.

A number of different matching techniques were tested, and nearest neighbour matching with common support was chosen because it created the closest matches. This process (called balancing) involved comparing the distribution of the propensity scores within the exposed and control groups, and also comparing the mean of each covariate in both groups. Ideally, the percentage difference in the means of the two groups (the percentage bias) should be less than $5 \%$. In this case, none of the matching techniques were quite able to achieve this (see Appendix 1, Table 2 for the selected approach). The matching approach was chosen before doing the final logistic regression, i.e. blind to the effect of the matching method on the outcome.

Finally, a simple logistic regression was carried out within the matched cases only between the six behavioural outcome variables and exposure to BIB to obtain the relevant odds ratios testing our hypotheses. A simple linear regression was carried out for birth weight.

\section{Testing the differential effect of mere measurement by socioeconomic status}

Mother's education was chosen as the best available indicator of socioeconomic status. Education was dichotomised into those educated up to GCSE level, and those 
with higher levels of education. Separate logistic regressions were then carried out stratified by mother's education, in order to compare results. Logistic regression was carried out using pairs matched on propensity score. Only outcomes showing the largest effects in the previous propensity score analysis were tested - any alcohol consumption and birth weight.

Significance levels for all analyses were two-sided and set at $5 \%$. Stata 12 was used, with additional user-written programmes psmatch2 [28] and pscore [29] for propensity score matching.

\section{Results}

\section{Summary statistics}

Table 1 shows summary statistics of our control and exposed groups. BIB P1s, who had not yet participated in the study, were very slightly older $(\mathrm{Z}=3.97, p<0.001, \mathrm{~d}=.20)$ and filled out their questionnaire earlier $(\mathrm{Z}=-12.29, p<0.001, \mathrm{~d}=.75)$ than BIB P2s.

\section{Propensity score matching}

Logistic regressions and balancing for propensity score matching are shown in appendix 1 . Nearest neighbour matching produced a sample size of 156 matched pairs $(n=312)$.

Table 2 shows the results of logistic regression between BIB P1s and BIB P2s, matched on propensity score. When restricting to women on their second pregnancy, the only significant finding was for any alcohol consumption where the odds of drinking was $65 \%$ less for women who had been exposed to BIB compared to those who had only just joined the cohort (odds ratio 0.35, 95\% CIs: 0.13, 0.92). The effect estimates of smoking and drinking five or more units of alcohol once a month or more were both in the direction of an improvement in health behaviours, and the effect estimate for birth weight also showed an increase. Effect estimates for vitamin D consumption and caffeine consumption were both marginally in the direction of a deterioration in health behaviours, though the effect sizes were very small (close to zero).

\section{Testing the differential effect of mere measurement by socioeconomic status}

The previous section addressed the first research question: Has being part of BIB influenced participants' health behaviour? This provided the basis for looking at the second question: Is the effect of participation in BIB on health moderated by socioeconomic status?

As many outcomes showed no significant change with exposure to BIB, just two outcomes were taken forward to test for mediation by socioeconomic status: any alcohol consumption (as it had shown a statistically significant
Table 1 Baseline comparison: Covariates by exposure to BIB

\begin{tabular}{|c|c|c|}
\hline & $\begin{array}{l}\text { BIB P1s (unexposed) } \\
N=1,411\end{array}$ & $\begin{array}{l}\text { BIB P2s (exposed) } \\
N=225\end{array}$ \\
\hline \multicolumn{3}{|l|}{ Age in years } \\
\hline Mean (SD) & $29.60(4.63)$ & $28.28(4.64)$ \\
\hline \multicolumn{3}{|l|}{ Ethnicity (\%) } \\
\hline White British & 32.24 & 28.89 \\
\hline Pakistani & 54.69 & 59.11 \\
\hline Other & 13.07 & 12.00 \\
\hline \multicolumn{3}{|l|}{ Education (\%) } \\
\hline$<5$ GCSEs & 24.11 & 24.44 \\
\hline 5 GCSES & 33.93 & 36.00 \\
\hline A-levels & 13.73 & 10.67 \\
\hline Higher than A-level & 20.27 & 24.44 \\
\hline Other & 7.97 & 4.44 \\
\hline $\begin{array}{l}\text { Questionnaire date } \\
\text { Mean (SD) }\end{array}$ & $659.98(384.71)$ & $999.48(237.66)$ \\
\hline \multicolumn{3}{|l|}{ Any alcohol (\%) } \\
\hline Yes & 53.82 & 33.33 \\
\hline No & 46.18 & 66.67 \\
\hline \multicolumn{3}{|c|}{ Alcohol - 5 units or more (\%) } \\
\hline Yes & 25.00 & 25.00 \\
\hline No & 75.00 & 75.00 \\
\hline \multicolumn{3}{|l|}{ Smoking (\%) } \\
\hline Yes & 15.13 & 12.50 \\
\hline No & 84.87 & 87.50 \\
\hline \multicolumn{3}{|l|}{ Vitamin D (\%) } \\
\hline Yes & 15.10 & 9.33 \\
\hline No & 84.90 & 90.67 \\
\hline \multicolumn{3}{|l|}{ Caffeine (\%) } \\
\hline Yes & 61.30 & 59.11 \\
\hline No & 38.70 & 40.89 \\
\hline $\begin{array}{l}\text { Birth weight (grams) } \\
\text { Mean (SD) }\end{array}$ & $3268.28(534.16)$ & $3315.04(496.00)$ \\
\hline
\end{tabular}

difference) and birth weight, as an objectively measured variable.

Table 3 presents the result of two bivariate regressions each stratified by education, using pairs matched on propensity score. Although both outcomes showed a larger effect estimate for those with higher education than for those with lower education, neither was statistically significant.

\section{Discussion}

This study did not provide conclusive evidence that a mere measurement effect consistently occurred in the BIB cohort study, or that it was moderated by socioeconomic status. Although some significant findings did 
Table 2 Odds ratios and coefficient for exposure to BIB, propensity score matched pairs

\begin{tabular}{lll}
\hline & Odds Ratio $(95 \% \mathrm{Cls})$ & $P$ value \\
\hline Smoking & $0.75(0.41,1.38)$ & 0.36 \\
Any alcohol & $0.35(0.13,0.92)$ & 0.03 \\
$\begin{array}{l}5 \text { or more units of alcohol } \\
\text { once a month or more }\end{array}$ & $0.24(0.03,2.20)$ & 0.21 \\
Vitamin D & $0.83(0.42,1.66)$ & 0.60 \\
Caffeine & $1.05(0.67,1.66)$ & 0.82 \\
& Coefficient $(95 \%$ Cls $)$ & $P$ value \\
Birth weight in grams & $41.95(-67.53,151.44)$ & 0.45 \\
\hline
\end{tabular}

emerge suggesting that further exploration of this topic is merited, the majority of tests showed no statistically significant effect.

1. Has being part of Born in Bradford improved participants' health behaviour?

Exposure to BIB was associated with a statistically significant improvement in health behaviours in only one of six regressions carried out - a significant reduction in the number of women reporting any alcohol consumption.

2. If so, has this effect been moderated by socioeconomic position? When cmstratifying by education, no significant effects were found either for any alcohol consumption, or for birth weight. However, effect estimates were larger for women of higher education, compared to women of lower education.

\section{Limitations}

\section{Non-randomisation}

As a non-randomised natural experiment, this study design is vulnerable to confounding based on noncomparability of the control and exposed groups. Another approach to seeing if taking part in BIB affected health behaviour is a before and after study, comparing first and second BIB pregnancies for the same women. Because the same women are in the control and exposed groups, many potential cofounders are avoided. Such a before and after design would not be a good way to demonstrate whether BIB had made a difference, as

Table 3 Propensity score matching: Odds ratio and coefficients for smoking, any alcohol consumption and birth weight by exposure to BIB, stratified by education, matched pairs

\begin{tabular}{lll}
\hline & \multicolumn{3}{l}{ Odds ratio by exposure to BIB $(95 \% \mathrm{Cls})$} \\
\hline \multirow{3}{*}{ Any alcohol } & 5 GCSEs or fewer & A-levels or higher \\
& $0.43(0.12,1.47)$ & $0.26[(0.05,1.28)$ \\
& Coefficient by exposure to BIB $(95 \% \mathrm{Cls})$ \\
Birth weight & $-39.25(-186.04,107.55)$ & $161.22(-69.14,391.58)$ \\
\hline
\end{tabular}

there would be no way to distinguish behavioural changes that would occur anyway with increased numbers of children from changes due to participation in BIB. Nevertheless, if BIB had improved behaviour it should show up with this design. This analysis was therefore carried out as a sensitivity test and is shown in Appendix 2.

No significant difference was found in any health behaviour between women's first and second pregnancy in BIB, although the effect sizes for both measures of alcohol use (OR 0.92 for any alcohol and 0.81 for 5 or more units of alcohol) indicate a non-significant reduction in alcohol consumption. A statistically significant difference in birth weight was identified, however. On their second BIB pregnancy, women had babies that were an average of $137.29 \mathrm{~g}$ larger than their first child within the BIB cohort, and this difference was statistically significant (95\% CIs 35.52, 239.07). This finding does not rule out the possibility that there could be some modest improvement as a result of exposure to BIB, which does not reach significance in individual behaviours, but does amount to an overall improvement in mother's health and therefore birth weight. However, the fact that these effect sizes are not large or significant, whereas our primary study design did show significance on one of the behavioural outcomes does suggest that those women who had two pregnancies within the cohort period may differ in unknown variables which are not possible to account for in propensity score matching.

\section{The trade-off between robustness and power}

Rather than adjusting for differences between the two groups, propensity score matching comes closer to imitating a randomised controlled trial by creating two groups that are as closely matched as possible, thereby only comparing observations that are similar enough to be comparable, based on known covariates [26, 30, 31]. This approach may also limit residual confounding; by only including in the analysis controls that have a similar propensity score, bias caused by any unknown variable that is a confounder and associated with one of the known covariates should be reduced.

However, the use of propensity score matching, as well as the restriction of the sample to second pregnancies involved a substantial loss of sample size and power.

There are some clues that a larger sample size may have been needed. Although only the regression testing for any alcohol consumption produced a significant finding, the estimated effect of exposure for BIB on smoking, consuming five or more units of alcohol once a month or more, and birth weight showed an improvement. The effect estimates for Vitamin D consumption and caffeine were in the direction of a deterioration, though both were close to zero. 
When testing for moderation by socioeconomic status, significance was lost on any alcohol consumption for more and less educated women, but the effect estimates for both any alcohol and birth weight indicated a larger improvement in health behaviours for more educated, compared to less educated women.

Sample size is particularly important for studies of mere measurement where effect sizes are likely to be small [4], and for exploring moderation by socioeconomic status, where a still larger sample size may be required to assess more subtle distributional effects.

\section{Outcome measures and fertility}

Both alcohol consumption [32] and smoking [33] are known to reduce fertility. This creates a potential bias if it results in women who drink and smoke with their first child being less likely to have another child within the four year BIB recruitment period. If this were the case, BIB P2s would have improved health behaviour not because of the effect of BIB, but because of selection bias between the exposed and unexposed groups, leading to a possible type 1 error. However, as identified above, selection bias cannot fully account for those significant findings which were identified as the differences at baseline were smaller than those found after the BIB P2s had participated.

\section{Recruitment of lower socioeconomic groups}

Any studies in which participation is conditional on agreeing to take part in a survey are open to participation bias. In particular, studies may struggle to recruit participants of lower socioeconomic status. Analysis comparing the BIB cohort with all other births at the maternity department in Bradford Teaching Hospital found that the cohort did have marginally lower representation from mothers living in more deprived areas [34] [Table 1]. In the case that there was a difference in take-up between those of lower and higher education, the exclusion of those with very low socio-economic status could lead to a type 2 error.

\section{Further research}

As we suggest above, there are theoretical reasons to expect that a mere measurement effect may be moderated by socioeconomic status. If this were the case, it would need to be taken into account in any long-term studies of health, and would also pose important ethical challenges for research studies, particularly those such as BIB that aim to decrease health inequalities. Although not conclusive, our findings suggest that this issue merits further study.

If such a question were to be incorporated into the design of a new research project, it could be designed as a nested RCT within an existing study, as described above in McCambridge and Kypri [5]. Such a design could overcome the challenges of selection bias found in this natural experiment and could easily incorporate an analysis of moderation with socioeconomic status. Ideally, such an analysis could incorporate more sensitive measures of socioeconomic status, including for example income, and explore how these different measures affected outcomes [35]. Crucially, the study should be powered not only to detect the small effect sizes expected from mere measurement, but also to detect differences between socioeconomic groups.

\section{Conclusion}

Although there are many studies showing small effects from mere measurement, research has so far failed to explore whether these effects are moderated by socioeconomic status. Overall, this study did not find sufficient evidence to conclude that mere measurement effect had occurred in the BIB cohort study, or that it had been moderated by socioeconomic status. However, one out of six of our analyses showed significant effect, and effect estimates suggested that participation in BIB may be associated with larger positive health effects for women with higher education. Research using designs with more comparable control groups and a larger sample size and are needed in order to explore potential moderation with socioeconomic status.

\section{Endnotes}

${ }^{1}$ The baseline questionnaire can be found at http:// www.borninbradford.nhs.uk/uploads/documents/questionaires/Born_in_Bradford_Mothers_Baseline_Questionnaire.pdf.

${ }^{2}$ Strictly the difference between BIB P1s and BIB P2s is a difference if gravidity rather than parity as BIB P2s have not yet had their second live birth within BIB. For simplicity however, the term parity is used throughout.

${ }^{3}$ Current NHS advice can be found at: http://www.nhs. uk/conditions/pregnancy-and-baby/pages/pregnancy-and -baby-care.aspx\#close.

\section{Appendix 1}

\section{Balancing for propensity score matching}

Table 4 presents the results of the logistic regression carried out in order to obtain the propensity scores. The outcome variable was exposure to $\mathrm{BIB}$, and the odds ratios indicate the odds of being a BIB P2 according to each covariate.

There were 225 BIB P2s on their second pregnancy. Only 156 of these BIB P2s were found appropriate matches due to only matching BIB P1s that were within the range of BIB P2s. Table 5 shows that after matching, the percentage bias was within $5 \%$ for all covariates except education which maintained a difference of $-12.1 \%$. Fig. 2 shows that the distribution of the propensity scores between BIB P1s and BIB P2s was adequately similar 
Table 4 Propensity score matching: Odds ratios for exposure to BIB

\begin{tabular}{lll}
\hline & $\begin{array}{l}\text { Adjusted Odds Ratios } \\
(95 \% \mathrm{Cls})^{\mathrm{a}}\end{array}$ & Adjusted $p$ value ${ }^{\mathrm{a}}$ \\
\hline $\begin{array}{l}\text { Age (per year increase) } \\
\text { Ethnicity }\end{array}$ & $1.01(0.96,1.07)$ & 0.74 \\
$\begin{array}{l}\text { White British } \\
\text { Pakistani }\end{array}$ & $\begin{array}{l}\text { Reference } \\
\text { Other }\end{array}$ & $0.96(0.58,1.58)$ \\
Education & $1.29(0.60,2.78)$ & 0.60 \\
$<5$ GCSEs & Reference & \\
5 GCSEs & $0.72(0.39,1.33)$ & 0.29 \\
A-levels & $0.81(0.36,1.84)$ & 0.61 \\
Higher than A-level & $0.63(0.32,1.25)$ & 0.19 \\
Other & $0.36(0.13,1.00)$ & 0.050 \\
Questionnaire date (per & $1.00(1.00,1.00)$ & 0.92 \\
day increase) & & \\
\hline
\end{tabular}

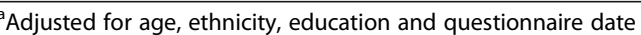

Table 5 Comparison of means of covariates between BIB P1s and BIB P2s, before and after matching on propensity score

\begin{tabular}{lllrr}
\hline & & \multicolumn{2}{c}{ Mean } & \% of bias \\
\cline { 3 - 4 } & & $\begin{array}{l}\text { BIB P2 } \\
\text { (exposed) }\end{array}$ & $\begin{array}{l}\text { BIB P1 } \\
\text { (unexposed) }\end{array}$ \\
\hline Age & Unmatched & 28.28 & 29.61 & -28.7 \\
& Matched & & 28.48 & -4.4 \\
Ethnicity & Unmatched & 1.83 & 1.80 & 4.2 \\
& Matched & & 1.81 & 3.5 \\
Education & Unmatched & 2.48 & 2.54 & -4.7 \\
& Matched & & 2.64 & -12.1 \\
Questionnaire & Unmatched & 999.48 & 661.77 & 105.7 \\
date & Matched & & 1012.6 & -4.1 \\
\hline
\end{tabular}

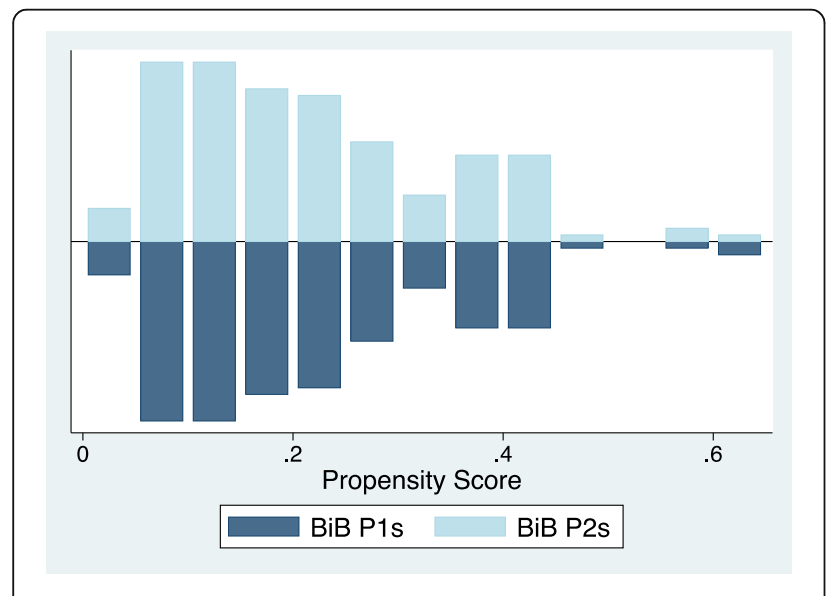

Fig. 2 Distribution of propensity scores by matched BIB P1s and BIB P2S

\section{Appendix 2}

\section{Before and after sensitivity test}

Table 6 presents regression analyses between women who participated in BIB twice in a before and after test. Their first pregnancy in the cohort acted as a control (BIB P1) while their second pregnancy acted as the intervention. Note that only women who had two pregnancies in the BIB cohort study were included in the control group.

Table 6 Before and after study of same women's BIB P1 and BIB P2

\begin{tabular}{lll}
\hline Outcome variables: & $\begin{array}{l}\text { Odds ratios for } \\
\text { exposure to BiB }\end{array}$ & Adjusted $p$ value \\
\hline Smoking & $0.98(0.56,1.71)$ & 0.95 \\
Any alcohol & $0.92(0.54,1.57)$ & 0.75 \\
5 or more units of alcohol & $0.81(0.35,1.88)$ & 0.62 \\
Vitamin D & $0.71(0.46,1.11)$ & 0.14 \\
Caffeine & $1.22(0.86,1.73)$ & 0.26 \\
Outcome variables & Coefficient for exposure & Adjusted $p$ value \\
Birth weight & to BlB & \\
\hline
\end{tabular}

\section{Abbreviations}

BIB: Born in Bradford cohort study; NHS: National health service; OR: Odds ratio; SES: Socio-economic status; SIDS: Sudden infant death syndrome

\section{Acknowledgements}

This study was funded by a studentship awarded to AQ from the Collaboration in Leadership for Applied Health Research and Care for Leeds, York and Bradford. This study has been made possible by the families in the Born in Bradford family cohort study, and to the help from staff working on the project, particularly Ann Barratt for providing information about Born in Bradford to the authors. Further acknowledgements are due to Noortje Uphoff for her help in accessing data to support revisions to the manuscript.

\section{Funding}

This study was funded by a studentship awarded to AQ from the Collaboration in Leadership for Applied Health Research and Care for Leeds, York and Bradford. The funding body played no role in design of the study and collection, analysis, and interpretation of data or in writing the manuscript.

\section{Availability of data and materials}

The datasets generated during and/or analysed during the current study are not publicly available although plans are in place to move towards open access in the near future. In the meantime, datasets are available from the corresponding author on reasonable request.

\section{Authors' contributions}

AQ participated in designing the study, carried out all analyses and drafted the manuscript. JRB provided input on statistical methods, in particular identifying the method of propensity score analysis, and helped draft the manuscript. JW helped to conceive of the study, participated in designing the study and commented on drafts of the manuscript. KEP helped to conceive of the study, participated in designing the study, identifying the appropriate analyses and helped draft the manuscript. All authors read and approved the final manuscript.

\section{Competing interests}

The authors declare that they have no competing interests. 


\section{Consent for publication}

Not applicable.

\section{Ethics approval and consent to participate}

Ethical approval for the data collection was granted by Bradford Research Ethics Committee (Ref 07/H1302/112).

All mothers were provided with an information sheet before recruitment which explained the importance of linking the data Born in Bradford collects to their NHS records in order to capture these health outcomes. It was further explained in detail by the research administrators at the time of recruitment. Written consent was then obtained from the mothers on behalf of themselves and their children. Born in Bradford staff also undertook regular focus group discussions with mothers to discuss their understanding of the consent they have given and to confirm their original decision.

\section{Author details}

'Department of Health Sciences, University of York, Heslington, York YO10 5DD, UK. ${ }^{2}$ Mental Health and Addiction Research Group (MHARG), Hull York Medical School and Department of Health Sciences, University of York, Heslington, York YO10 5DD, UK. ${ }^{3}$ Bradford Institute for Health Research, Temple Bank House, Bradford Royal Infirmary, Duckworth Lane, Bradford BD9 $6 R J$, UK.

Received: 27 July 2015 Accepted: 16 January 2017

Published online: 25 January 2017

\section{References}

1. Murray M, Swan AV, Kiryluk S, Clarke GC. The Hawthorne effect in the measurement of adolescent smoking. J Epidemiol Community Health. 1988;42(3):304-6.

2. French DP, Sutton S. Reactivity of measurement in health psychology: How much of a problem is it? What can be done about it? Br J Health Psychol. 2010;15(3):453-68

3. Godin G, Sheeran P, Conner M, Germain M. Asking questions changes behavior: mere measurement effects on frequency of blood donation. Health Psychol. 2008;27:179-84.

4. Rodrigues, A. M., O'Brien, N., French, D. P., Glidewell, L., \& Sniehotta, F. F. The Question-Behavior Effect: Genuine Effect or Spurious Phenomenon? A Systematic Review of Randomized Controlled Trials With Meta-Analyses. Health Psychology. 2014; doi: 10.1037/hea0000104.

5. McCambridge J, Kypri K. Can simply answering research questions change behaviour? Systematic review and meta analyses of brief alcohol intervention trials. PLoS One. 2011;6(10):e23748.

6. van Sluijs EMF, et al. Physical activity measurements affected participants' behavior in a randomized controlled trial. J Clin Epidemiol. 2006:59(4):404-11.

7. Castonguay L, Barkham M, Lutz W, McAleavey A. Practice-Oriented Research: Approaches and Applications. In M. J. Lambert (Ed.). Handbook of Psychotherapy and Behavior Change, 6th edition. New York: Wiley; 2013. p. 85-133.

8. Fung $\mathrm{CH}$, Hays RD. Prospects and challenges in using patient-reported outcomes in clinical practice. Qual Life Res. 2008;17(10):1297-302.

9. Valderas JM, et al. The impact of measuring patient-reported outcomes in clinical practice: a systematic review of the literature. Qual Life Res. 2008;17(2):179-93.

10. Shimokawa K, Lambert MJ, Smart DW. Enhancing treatment outcome of patients at risk of treatment failure: meta-analytic and mega-analytic review of a psychotherapy quality assurance system. J Consult Clin Psychol. 2010;78(3):298-311.

11. Durham CJ, McGrath LD, Burlingame GM, Schaalje GB, Lambert MJ, Davies DR. The effects of repeated administrations on self-report and parent-report scales. J Psychoeduc Assess. 2002;20(3):240-57.

12. Greenhalgh J, Long AF, Flynn R. The use of patient reported outcome measures in routine clinical practice: lack of impact or lack of theory? Soc Sci Med. 2005;60(4):833-43.

13. Strauss BM, et al. Benefits and challenges in practice-oriented psychotherapy research in Germany: The TK and the QS-PSY-BAY projects of quality assurance in outpatient psychotherapy. Psychother Res. 2015;25(1):32-51.

14. Christakis NA, Fowler JH. The collective dynamics of smoking in a large social network. N Engl J Med. 2008;358(21):2249-58.
15. Arblaster L, Lambert M, Entwistle V, Forster M, Fullerton D, Sheldon T, et al. A systematic review of the effectiveness of health service interventions aimed at reducing inequalities in health. J Health Serv Res. 1996;1(2):93-103.

16. Pickett KE, Luo Y, Lauderdale DS. Widening social inequalities in risk for sudden infant death syndrome. Am J Public Health Res. 2005;95(11): 1976-81.

17. Graham H. Smoking prevalence among women in the European Community 1950-1990. Soc Sci Med. 1996;43(2):243-54.

18. Marmot MG. Status syndrome: how your social standing directly affects your health and life expectancy. London: Bloomsbury; 2004.

19. Spencer N. Behaving badly? Smoking and the role of behaviour change in tackling health inequalities. Challenging health inequalities: From Acheson to 'Choosing Health. 2007. p. 157-74.

20. Ferguson J, et al. The English smoking treatment services: one-year outcomes. Addiction. 2005;100(s2):59-69.

21. Windham GC, et al. Prenatal active or passive tobacco smoke exposure and the risk of preterm delivery or low birth weight. Epidemiology. 2000;11(4):427-33.

22. Jaddoe $\mathrm{WW}$, et al. Moderate alcohol consumption during pregnancy and the risk of low birth weight and preterm birth. The generation R study. Ann Epidemiol. 2007;17(10):834-40.

23. Valero de Bernabé J, et al. Risk factors for low birth weight: a review. Eur J Obstet Gynecol Reprod Biol. 2004;116(1):3-15.

24. Mannion CA, Gray-Donald K, Koski KG. Association of low intake of milk and vitamin D during pregnancy with decreased birth weight. Can Med Assoc J. 2006;174(9):1273-7.

25. Thorne-Lyman A, Fawzi WW. Vitamin D during pregnancy and maternal, neonatal and infant health outcomes: a systematic review and metaanalysis. Paediatr Perinat Epidemiol. 2012;26(s1):75-90.

26. Guo S, Fraser MW. Propensity score analysis : statistical methods and applications. Los Angeles. London: Sage; 2010.

27. Rosenbaum PR, Rubin DB. The central role of the propensity score in observational studies for causal effects. Biometrika. 1983;70(1):41-55.

28. Leuven, E., \& Sianesi, B. PSMATCH2: Stata module to perform full Mahalanobis and propensity score matching, common support graphing, and covariate imbalance testing. Statistical Software Components. 2014

29. Becker SO, Ichino A. Estimation of average treatment effects based on propensity scores. Stata J. 2002;2(4):358-77.

30. Austin PC. An introduction to propensity score methods for reducing the effects of confounding in observational studies. Multivariate Behav Res. 2011;46(3):399-424.

31. Cummings P. Propensity scores. Arch Pediatr Adolesc Med. 2008;162(8):734-7.

32. Eggert J, Theobald H, Engfeldt P. Effects of alcohol consumption on female fertility during an 18-year period. Fertil Steril. 2004;81(2):379-83.

33. Augood C, Duckitt K, Templeton AA. Smoking and female infertility: a systematic review and meta-analysis. Hum Reprod. 1998;13(6):1532-9.

34. Wright, J., et al. Cohort profile: The Born in Bradford multi-ethnic family cohort study. Int Journal Epidemiol. 2012; Doi:10.1093/ije/dys112 [doi] [published Online First: Epub Date]

35. Braveman PA, Cubbin C, Egerter S, Chideya S, Marchi KS, Metzler M, et al. Socioeconomic status in health research: one size does not fit all. JAMA. 2005:294(22):2879-88.

\section{Submit your next manuscript to BioMed Central and we will help you at every step:}

- We accept pre-submission inquiries

- Our selector tool helps you to find the most relevant journal

- We provide round the clock customer support

- Convenient online submission

- Thorough peer review

- Inclusion in PubMed and all major indexing services

- Maximum visibility for your research

Submit your manuscript at www.biomedcentral.com/submit 